\title{
Simple linear viscoelastic models of dilute solutions of polymer molecules and emulsion droplets
}

\author{
J. Mellema, C. Blom and J. Beekwilder \\ Rheology Group, Department of Applied Physics, Twente University of Technology, Enschede (The Netherlands)
}

\begin{abstract}
The complex viscosity of microemulsions shows relaxation processes of which the largest relaxation time is about $10^{-5} \mathrm{~s}$ or less. This time can be attributed to relaxation of stresses in the surface of emulsion droplets pertaining to interfacial tension. Superimposed on a spherical droplet surface shape fluctuations can occur due to thermal energies. Our aim is to show the influence of thermal shape fluctuations on the complex viscosity of emulsions. The method used in the derivation has also been applied to inflexible rods to demonstrate its feasibility by showing the formal rheological equivalence of in length thermally fluctuating rods and Rouse's simple model of polymers. The emulsion results have been applied to a dilution series of a non-ionic microemulsion.
\end{abstract}

Key words: Polymer, emulsion, complex viscosity, linear viscoelasticity, relaxation time

\section{Introduction}

In the fifties fundamental research on the linear viscoelastic behaviour of (semi)dilute polymer solutions got great impetus to which Rouse's [1] and Zimm's [2] theoretical work and the possibility of the measurement of the complex modulus of these systems significantly contributed.

The development of fundamental research of the linear viscoelastic behaviour of a counterpart of polymer solutions in dispersion rheology is quite different. Though the first theories on e.g. dilute emulsions given by Oldroyd $[3,4]$ also appeared in the fifties they were neither accompanied nor closely followed by experimental corroboration. The reason for this can be attributed to an experimental difficulty concerning ordinary emulsions. The largest relaxation time $\tau$ for an ordinary emulsion droplet with an interfacial tension $\gamma$ can be estimated as $\tau \approx a \eta / \gamma$, where $a$ is the radius of the droplet and $\eta$ an effective viscosity close to the viscosity of the emulsion. For an ordinary emulsion $a \approx 10^{-5} \mathrm{~m}, \gamma \approx 10^{-2} \mathrm{~N} / \mathrm{m}$ and $\eta \approx 10^{-3} \mathrm{~Pa} \mathrm{~s}$. This gives $\tau \approx 10^{-6} \mathrm{~s}$.

In an harmonically oscillating shear experiment with angular frequency $\omega$ the reciprocal wave number is

$$
\frac{\lambda}{2 \pi}=\frac{1}{\omega} \sqrt{\frac{2}{\varrho} \frac{\left(G^{\prime 2}+G^{\prime 2}\right)}{\sqrt{\left(G^{\prime 2}+G^{\prime 2}\right)+G^{\prime}}}},
$$

with $\lambda$ the wave length.
Here $G^{\prime}$ and $G^{\prime \prime}$ are the real and imaginary parts of the complex modulus respectively and $\varrho$ is the density of the liquid. At $\omega=1 / \tau$ this length is at low volume concentrations about $10^{-6} \mathrm{~m}$. Consequently no bulk properties can be measured for ordinary emulsions in a dynamic shear experiment.

In the seventies the situation changed. On large scale information on so called microemulsions became available. If droplets of these emulsions are almost spherical they have a radius between $10^{-8}$ and $10^{-6} \mathrm{~m}$ while their interfacial tension is probably small $\left(10^{-4}-10^{-7}\right.$ $\mathrm{N} / \mathrm{m}$ ). In addition it turned out to be possible to make emulsions with a radius of about $5 \cdot 10^{-8} \mathrm{~m}$ [5]. In the mean time adequate apparatus development [6] enhanced the possibilities for the investigation of emulsions. As well for emulsions with small radius [5] as for microemulsions $[7,8]$ corroborations could be given for the notion of linear viscoelastic behaviour of emulsions due to interfacial tension effects. A striking feature of some microemulsion results in particular [7] was that the complex modulus showed more than two relaxation times which could not be accounted for by Oldroyd's theories. There may be several explanations for this phenomenon. In this paper the concept of the influence of thermal fluctuations of the droplet surfaces is pursued. As may be envisaged a complete theory cannot be given easily. A method to derive the complex modulus $G^{*}$ is proposed. It incorporates a number of essential aspects which would also appear in a 
sophisticated theory. The feasibility of the method has been demonstrated in section 3 for a dilute system of inflexible rods which can be mapped onto a simple (Rouse) model for dilute polymer solutions. The application of the method to emulsions reveals the possible existence of in principle infinite normal modes. The relaxation strengths of the modes are derivable from the stored elastic energies. The present results are valid for infinite dilution and contain as a special case the behaviour given by Oldroyd [3]. Assuming that the normal modes of the droplet surfaces are also present at finite concentration, the formal behaviour at finite concentration can be given with two additional parameters.

\section{Method}

As representative for linear viscoelastic behaviour in this paper the complex shear viscosity or complex shear modulus is considered. With aid of interrelations among the linear viscoelastic functions other representations can be derived [9]. The reflections are restricted to liquid systems which are Newtonian at infinitely long time scale and are still viscous at infinitely short time scale. Then the complex shear modulus $G^{*}$ and the complex viscosity $\eta^{*}$ are given by [10]:

$$
\begin{aligned}
G^{*} & =i \omega \eta_{\infty}+\sum_{l} G_{l} \frac{i \omega \tau_{l}}{1+i \omega \tau_{l}} \equiv i \omega \eta^{*} \\
& =i \omega\left[\eta_{\infty}+\sum_{l} \frac{\eta_{l}}{1+i \omega \tau_{l}}\right] .
\end{aligned}
$$

In this formula $\omega$ is the angular frequency, $\eta$ represents a viscosity, $\tau_{l}$ a relaxation time, and $G_{l}$ a relaxation strength.

The first assumption is that in the fluid constituents (solvent, solutes) can be discerned to which well defined properties are assigned which determine the quantities $\eta_{\infty}, G_{l}$ and $\tau_{l}$. Next it is assumed that the motions of solutes can be decomposed in modes of motion which are specific for the configurations of solute properties and elastic and viscous properties involved. As these modes of motion are most conveniently expressed in normal coordinates (see e.g. [11]) they are sometimes called normal modes.

Due to thermal energies the configuration of a solute is such that it fluctuates statistically about some equilibrium configuration. On the basis of the modes of motion one can calculate $\tau_{l}$. The relaxation strength $G_{l}$ is calculated by means of an elastic energy consideration of the solute configuration in a normal mode. It involves two steps: the calculation of the elastic energy of a normal mode for $\omega \rightarrow \infty$ and the relationship between the microscopic stored elastic energy and the macroscopic stored energy. The viscosity $\eta_{\infty}$ for $\omega \rightarrow \infty$ is the limit viscosity when all the relaxation processes (in case of a finite number) are fully excited. Its calculation is a well known problem and is not part of this paper.

\section{Inflexible rods}

\subsection{Polymer model mapped onto an inflexible rod model}

Firstly a polymer model is outlined. The polymer solution consists of polymer molecules dissolved in a
Newtonian fluid with viscosity $\eta_{s}$. There are $n$ molecules per unit volume each consisting of $h N$ monomers. The molecules can be thought to be divided in $N$ submolecules of $h$ monomers chosen such that the end to end orientation of a submolecule is independent of the others. The number $n$ is so small that the linear viscoelastic properties can be considered to be the sum of the single molecule effects. In addition the submolecules have no interaction with each other neither by volume nor hydrodynamically. The solute molecules are in thermal equilibrium with their surroundings. As a consequence a part of the polymer molecule between two points sufficiently far apart acts as a Gaussian spring with spring constant

$$
\frac{3 k T}{p b^{2}}
$$

if $p$ is the number of submolecules between the points and $p b^{2}$ is the average of the square of the distance between the points. Each part of the molecule is statistically fluctuating in conformation and position in space. A submolecule possesses a friction coefficient $f_{0}$ which is defined by:

$$
F=f_{0} \frac{\partial \xi}{\partial t} \text {. }
$$

Here $\partial \xi / \partial t$ is the relative velocity of the submolecule with respect to the undisturbed applied flow at the position of the submolecule and $F$ the force needed to maintain this velocity.

In first approximation such a molecule can be conceived as a one-dimensional inflexible rod with length $L$ along the $z$-axis with an elastic constant $K$ per unit length defined by

$$
\sigma=K \frac{\partial \xi}{\partial z}
$$

and a friction coefficient $\lambda$ per unit length defined analogously as $f_{0}$. In (5) $\sigma$ is the stress, $z$ the coordinate along the rod and $\xi$ the displacement at $z$ along the rod. This model is identical to that of Gross and Fuoss [12] who demonstrated that the relationship between $\sigma$ and a harmonic deformation $\partial \xi / \partial z$ is of the same form as the linear viscoelastic behaviour of a polymer solution according to Rouse [1]. The equation of motion (inertia terms neglected) which apply for waves in such a rod is

$$
\frac{\partial^{2} \xi}{\partial z^{2}}=q^{2} \frac{\partial \xi}{\partial t} \quad \text { with } \quad q^{2}=\frac{\lambda}{K} .
$$

Due to thermal motion displacement fluctuations can occur along the inflexible rod. The displacement $\xi$ at 
position $z_{0}$ and time $t$ can be given by

$$
\xi\left(z_{0}, t\right)=\sum_{l} A_{l}(t)\left[\cos b_{l} z_{0}+B_{l}(t) \sin c_{l} z_{0}\right]
$$

because the sine and cosine functions constitute a complete set of orthonormal functions. If the thickness of the rod is taken infinitely small no forces can be executed at the end points $(\partial \xi / \partial z=0)$ then eq. (7) reduces to

$$
\xi\left(z_{0}, t\right)=\sum_{l} A_{l}(t) \cos \left(\frac{l \pi}{L} z_{0}\right) .
$$

The coefficients $A_{l}$ can be considered as the normal coordinates of the modes of the rod. When the rod is deformed in a pure straining motion with a rate of strain given by

$$
D=\left[\begin{array}{rrr}
-\frac{\dot{\varepsilon}}{2} & 0 & 0 \\
0 & -\frac{\dot{\varepsilon}}{2} & 0 \\
0 & 0 & +\dot{\varepsilon}
\end{array}\right]
$$

the shape of a rod along the $z$-axis is in the first approximation given by

$$
z=\left[z_{0}+\sum_{l} A_{l} \cos \left(\frac{l \pi}{L} z_{0}\right)\right]\left(1+\varepsilon^{\prime}\right)
$$

where $\varepsilon^{\prime}$ is proportional to the applied strain $\varepsilon$. Thus

$$
\begin{aligned}
z & =z_{0}\left(1+\varepsilon^{\prime}\right)+\sum_{l} A_{l}\left(1+\varepsilon^{\prime}\right) \cos \left(\frac{l \pi}{L} z_{0}\right) \\
& =z_{0}\left(1+\varepsilon^{\prime}\right)+\sum_{l} A_{l}^{\prime} \cos \left(\frac{l \pi}{L} z_{0}\right) .
\end{aligned}
$$

In a harmonically oscillating flow $\varepsilon^{\prime}$ will depend on the angular frequency $\omega$ and be different for each $l$ if $\omega$ is finite. For $\omega \rightarrow \infty$ each part of the rod moves affine with the flow in first approximation. Thus $\varepsilon^{\prime}(\omega \rightarrow \infty)=\varepsilon$ and the viscosity $\eta_{\infty}$ of the solution with infinitely thin rods for $\omega \rightarrow \infty$ will be the viscosity $\eta_{s}$ of the solvent.

\subsection{Relaxation times of the polymer normal modes}

To find the characteristic times with which excited modes relax one can apply in a thought experiment a deformation $A_{l}^{\prime}(t) \cos \left(\frac{l \pi}{L} z\right)$ at time $t=t_{0}$ and then calculate the relaxation time. When this shape is substituted in eq. (6) one finds

$$
-\left(\frac{l \pi}{L}\right)^{2} A_{l}^{\prime}(t)=q^{2} \frac{d A_{l}^{\prime}(t)}{d t}
$$

which has a solution

$$
A_{l}^{\prime}(t)=A_{l}^{\prime}\left(t_{0}\right) \exp \left[-\left(t-t_{0}\right) /\left(\frac{q L}{l \pi}\right)^{2}\right]
$$

and gives as the relaxation time

$$
\tau_{l}=\left(\frac{q L}{l \pi}\right)^{2} .
$$

Now the mapping of the parameters of these times to that of the polymer molecule raises a problem which is due to the Gaussian character of the polymer spring. The spring constant between two points of a polymer is not independent of the choice of the points (see (3)). The force direction in the rod is in all modes either anti-symmetric or point symmetric with respect to the centre of the rod. This implies that the deformation pattern is identical in each half of the rod. Transferring this fact to the case of the polymer molecule the relevant spring constant is completely determined by the half of the molecules. Consequently the relevant spring constant of the molecule to be mapped on $K / L$ is

$$
\frac{3 k T}{\frac{1}{2} N b^{2}}=\frac{K}{L} \text {. }
$$

The friction is unequivocally mapped as

$$
f_{0} N=\lambda L \text {. }
$$

Consequently it follows that the relaxation times of the polymer molecule are with eqs. $(6,14-16)$

$$
\tau_{l}=\frac{N^{2} b^{2} f_{0}}{6 \pi^{2} k T} \frac{1}{l^{2}} .
$$

According to the deformation in (11), however, there is the possibility of another process. It is pertinent to the elongation of the rod

$$
z=z_{0}\left(1+\varepsilon^{\prime}\right) .
$$

The characteristic time of such a deformation cannot exist in a polymer because it is impossible to apply a homogeneous elongation along a polymer molecule. It can be demonstrated to be equivalent to the relaxation process of a Hookean dumbell (see e.g. [13], p. 490 with $2 \xi=\lambda L$ and $K / L=H$ ).

\subsection{Determination of the polymer relaxation strengths $G_{l}$}

The elastic energy stored at time $t$ in a normal mode while the fluid does not flow is given by

$$
W_{l}(t)=\frac{1}{2} \frac{K}{L} A_{l}^{2}(t)\left(\frac{l \pi}{L}\right)^{2} .
$$


In a harmonically pure straining flow if the rod lies along the $z$-axis it becomes for $\omega \rightarrow \infty\left(\varepsilon_{\infty}^{\prime}=\varepsilon\right)$

$$
W_{l}^{\prime}(t)=\frac{1}{2} \frac{K}{L}\left[A_{l}(t)(1+\varepsilon)\right]^{2}\left(\frac{l \pi}{L}\right)^{2}
$$

The coefficients $A_{l}(t)$ are of thermal origin. The equipartition law allows to assign to each mode averaged over an infinite number of units a specific thermal energy. Here one deals again with the problem of the mapping of the vibration characteristics of the rod to those of a polymer. In section 3.2 it was made clear that for the dynamic behaviour the properties of the half of the molecule are relevant. Between the centre and an end of the molecule a Gaussian spring acts, to which to each mode of motion a thermal energy of $\frac{3}{2} k T$ should be assigned because of its three-dimensional character. Apparently averaged over all the particles for the fluid at rest it holds that

$$
\left\langle W_{l}\right\rangle=\frac{1}{2} \frac{K}{L}\left\langle A_{l}^{2}(t)\right\rangle\left(\frac{l \pi}{L}\right)^{2}=3 k T .
$$

If the orientations of the rods in space are randomly distributed the orientation averaged squared strain is

$$
\overline{\varepsilon^{2}}=\frac{1}{3}\left(\varepsilon^{2}+\frac{\varepsilon^{2}}{4}+\frac{\varepsilon^{2}}{4}\right)=\frac{1}{2} \varepsilon^{2} .
$$

The energy $W_{l}^{\prime}(t)$ is averaged over all rods and their directions. The linear term in $\varepsilon^{\prime}$ represents the asymmetry in energy for a predeformed rod when deformed with $1+\varepsilon$ and $1-\varepsilon$ which averages out in a harmonically oscillating flow. This term is omitted here and that is consistent with the assumption that macroscopically the material is not predeformed. Thus

$$
\left\langle W_{l}^{\prime}(t)\right\rangle=\frac{1}{2} \frac{K}{L}\left\langle A_{l}^{2}(t)\right\rangle\left(1+\frac{1}{2} \varepsilon^{2}\right)\left(\frac{l \pi}{L}\right)^{2} .
$$

Using (21) it follows that the extra stored energy due to the flow for one molecule in each mode if $\omega \rightarrow \infty$ is

$$
\frac{3}{2} k T \varepsilon^{2} .
$$

Macroscopically the stored energy in a pure straining motion per mode per molecule is

$$
\frac{3}{2} G_{l} \varepsilon^{2} \frac{1}{n} \text {. }
$$

Thus

$$
G_{l}=n k T .
$$

\subsection{Discussion of the polymer solution results}

According to the previous sections the complex modulus $G^{*}$ of a polymer is given by

$$
G^{*}=i \omega \eta^{*}=i \omega \eta_{s}+\sum_{l} G_{l} \frac{i \omega \tau_{l}}{1+i \omega \tau_{i}}
$$

with $G_{l}=n k T$ and

$$
\tau_{l}=\frac{f_{0} N^{2} b^{2}}{6 \pi^{2} k T} \frac{1}{l^{2}} \quad(l \geqq 1) .
$$

This modulus is identical to the approximation for large $N$ derived by Rouse. The seeming primitivity of the rod model suggests that it may only be convenient to derive the results of the simple bead-spring model of Rouse. Mijnlieff [14], however, has demonstrated that subtle reasoning can extend it, e.g. taking into account the non-ideal statistics and hydrodynamic interactions.

\section{Dilute emulsion}

\subsection{Model}

The emulsion is conceived as composed of two Newtonian fluids with viscosity $\eta_{1}$ and $\eta_{2}$. The fluids in contact with each other exhibit a constant interfacial tension $\gamma$. The fluid with viscosity $\eta_{2}$ is dispersed in the other fluid as droplets which equilibrium shapes are spherical. They are monodisperse with radius $a$ and take up a volume fraction $\phi$ which is so small that the linear viscoelastic properties can be considered to be the sum of the single particle effects (no interaction effects). The interface between the liquids is assumed to be one system with two geometrical degrees of freedom. The interfacial energy of the spherical equilibrium shape is $4 \pi a^{2} \gamma$. If the interface is in thermal equilibrium with its surroundings fluctuating deviations from the spherical shape will occur. Using polar coordinates $\theta$ and $\phi$ its shape at time $t$ can be given by

$$
R^{\prime}(\theta, \phi, t)=a\left[1+\sum_{l, m} A_{l m}(t) Y_{l m}(\theta, \phi)\right]
$$

because the spherical harmonics $Y_{l m}$ constitute a complete (orthonormal) set of functions. In (29) $m=$ $-l,-l+1, \ldots, l$ and for the complex conjugate $A_{l m}^{*}$ of $A_{l m}$ it holds that $A_{l m}^{*}=(-1)^{m} A_{l-m}$. The condition for (29) to be valid is that the shape $R^{\prime}(\theta, \phi, t)$ is a single valued function of its parameters.

For small deformations the extra interfacial tension energy due to the fluctuations is given by (see e.g. [15])

$$
W_{\gamma, l, m}(t)=\frac{1}{2} \gamma a^{2} \sum_{l, m}(l-1)(l+2) A_{l m}(t) A_{l m}^{*}(t) .
$$


Note that in this approximation $W_{\gamma, 1, m}=0$. The coefficients $A_{l m}$ can be considered as the normal coordinates of the motion of the interface. According to the equipartition law a thermal equilibrium, the value for each independent mode averaged over all the droplets should be equal to $\frac{1}{2} k T$, in formula

$$
\left\langle W_{,, l, m}\right\rangle=\frac{1}{2} \gamma a^{2}(l-1)(l+2)\left\langle A_{l m}(t) A_{l m}^{*}(t)\right\rangle=\frac{1}{2} k T .
$$

In a pure straining motion, which has in polar coordinates the $Y_{2,0}$-symmetry, eq. (9), one expects in first approximation that the new shape will be given by

$R(\theta, \phi, t)$

$$
=a\left\{1+\sum_{l, m}\left[A_{l m}(t) Y_{l m}(\theta, \phi)\left(1+\varepsilon_{l m}^{\prime} Y_{20}(\theta, \phi)\right)\right]\right\}
$$

where $\varepsilon_{l m}^{\prime}$ is proportional to the amplitude of the applied strain $\varepsilon$. Just like for the polymer in a harmonically oscillating flow $\varepsilon_{l m}^{\prime}$ will depend on the angular frequency $\omega$ and be different for each $l m$ if $\omega$ is finite.

From eq. (32) follows that a flow field with $Y_{20}$ symmetry can change the magnitude of the normal coordinates. Mathematically, the new shape can be written as

$$
\begin{aligned}
\mathrm{R}(t)=a\left\{1+\varepsilon_{20}^{\prime} Y_{20}+\sum_{l, m} A_{l m}(t) Y_{l m}\right. & \begin{array}{l}
\text { holding for the equilibrium shape at } \\
\text { from (36) the left-hand side goes to }
\end{array} \\
& \left.\left.+\sum_{l, m}\left[\sum_{k^{\prime}=|l-2|}^{l+2} A_{l m}(t) \varepsilon_{l m}^{\prime}\left(\frac{(2 l+1) 5}{4 \pi\left(2 k^{\prime}+1\right)}\right)^{1 / 2} C\left(l 2 m o \mid l 2 k^{\prime} m\right) C\left(l 200 \mid l 2 k^{\prime} 0\right) Y_{k^{\prime} m}\right)\right]\right\}
\end{aligned}
$$

or

$$
\begin{aligned}
R(t)=a & \left\{1+\varepsilon_{20}^{\prime} Y_{20}+\sum_{l, m}\left[A_{l m}(t)+\sum_{k^{\prime}=|l-2|}^{l+2}\left\{A_{k^{\prime} m}(t)\left(\frac{\left(2 k^{\prime}+1\right) 5}{4 \pi(2 l+1)}\right)^{1 / 2} \varepsilon_{l m}^{\prime}\right.\right.\right. \\
& \left.\left.\left.\cdot C\left(k^{\prime} 2 m 0 \mid k^{\prime} 2 l m\right) C\left(k^{\prime} 200 \mid k^{\prime} 2 l 0\right)\right\}\right] Y_{l m}\right\} \equiv a\left(1+\sum_{l, m} A_{l m}^{\prime}(t) Y_{l m}\right),
\end{aligned}
$$

when $C$ is a Clebsch-Gordan coefficient (see e.g. [16] p. 513). In these summations the breathing mode with $l=0$ is not included because incompressibility of the droplet is assumed. In addition $A_{1 m}(t)$ is undetermined in eq. (31) and consequently it cannot be considered in the scope of the present paper: in eq. (34) $l \geqq 2$ and $k^{\prime} \geqq 2$. The detection of the flow field changes can be imagined as follows. The macroscopic stress tensor $\langle\boldsymbol{\sigma}\rangle$, for the emulsion can be given by

$$
\begin{aligned}
& \langle\boldsymbol{\sigma}\rangle=\text { (pressure term) }+2 \eta_{1}\langle\boldsymbol{D}\rangle \\
& \quad+\lim _{V \rightarrow \infty} \frac{1}{V}\left(\eta_{2}-\eta_{1}\right) \int_{\partial V_{p}}(\boldsymbol{u} \boldsymbol{n}+\boldsymbol{n} \boldsymbol{u}) d S+\frac{1}{V} \int_{\partial V_{\boldsymbol{p}}} \gamma \boldsymbol{U}_{\mathrm{II}} d S
\end{aligned}
$$

(see [17]). Here $\langle\boldsymbol{D}\rangle$ is the macroscopic rate of strain tensor, $V$ is a volume, $\partial V_{p}$ is the surface of the droplets
Apparently for $\omega \rightarrow 0$ the sphere is hardly deformed by the flow and $\varepsilon_{l m}^{\prime} \rightarrow 0$.

Because of the orthogonality of the $Y_{l m}$ 's condition (36) applies for each $Y_{l m}$. In spite of the orthogonality the $A_{l m}$ 's can change due to the coupling with the applied flow field. These changes are detectable because the applied field is disturbed by the droplet according to (32).

\subsection{The viscosity $\eta_{\infty}$ at inifinite angular frequency}

According to eqs. (35) and (36) and the reasoning in section 4.1 the stress tensor at infinite angular fre- 
quency can be given by:

$$
\begin{aligned}
\langle\boldsymbol{\sigma}\rangle_{\omega \rightarrow \infty} & =(\text { pressure term })+2 \eta_{1}\langle\boldsymbol{D}\rangle \\
& +\lim _{V \rightarrow \infty} \frac{1}{V}\left(\eta_{2}-\eta_{1}\right) \int_{\partial V_{p}}(\boldsymbol{u} \boldsymbol{n}+\boldsymbol{n} \boldsymbol{u}) d S .
\end{aligned}
$$

Apparently the droplet contribution to $\eta_{\infty}$ should be proportional to $\eta_{2}-\eta_{1}$. As far as we know no exact expression for $\eta_{\infty}(\gamma=0)$ exists. It is possible, however, to give so-called Hashin-Shtrikman bounds [17], which
Here $\boldsymbol{u}$ is the velocity and $p$ the pressure. The applied field is rotationally symmetric. Accordingly the vortex lines for a sphere placed in this flow are circles about the axis of $z$. Mathematically this is expressed by $\boldsymbol{r} \cdot \operatorname{rot} \boldsymbol{u}=0$ where $\boldsymbol{r}$ is the radius vector. Since the $A_{l m}$ 's should be small for the studied droplets the vector lines are close to circles about the axis of $z$. Without further justification the vortex lines are taken as circles subsequently. Thus the solution of (40) with the extra condition $\boldsymbol{r} \cdot \operatorname{rot} \boldsymbol{u}=0$ is given by

$$
\begin{aligned}
& u\langle r\rangle=\left[\frac{l}{2(2 l+3)} A_{l} r^{l+1}+\frac{(l+1)}{2(2 l-1)} B_{l} r^{-l}+l r^{l-1} C_{l}+(-l-1) D_{l} r^{-l-2}\right] Y_{l m}(\theta, \phi), \\
& u\langle\theta\rangle=\left[\frac{l+3}{2(2 l+3)(l+1)} A_{l} r^{l+1}+\frac{(2-l)}{2 l(2 l-1)} B_{l} r^{-l}+r^{l-1} C_{l}+D_{l} r^{-l-2}\right] Y_{l m}^{\theta}(\theta, \phi), \\
& \sigma\langle r r\rangle=\eta\left[\frac{l^{2}-l-3}{2 l+3} A_{l} r^{l}+\frac{\left(l^{2}+3 l-1\right)}{(1-2 l)} B_{l} r^{-l-1}+2 l(l-1) r^{l-2} C_{l}+2(l+1)(l+2) D_{l} r^{-l-3}\right] Y_{l m}(\theta, \phi), \\
& \sigma\langle r \theta\rangle=\eta\left[\frac{l(l+2)}{(l+1)(2 l+3)} A_{l} r^{l}+\frac{(l-1)(l+1)}{l(2 l-1)} B_{l} r^{-l-1}+(2 l-2) r^{l-2} C_{l}+(-2 l-4) D_{l} r^{-l-3}\right] Y_{l m}^{\theta}(\theta, \phi) .
\end{aligned}
$$

are for $\eta_{1} \leqq \eta_{2}$

$$
\begin{aligned}
\eta_{1} \frac{3 \eta_{1}+2 \eta_{2}+3\left(\eta_{2}-\eta_{1}\right) \phi}{3 \eta_{1}+2 \eta_{2}-2\left(\eta_{2}-\eta_{1}\right) \phi} \\
\leqq \eta_{\infty} \leqq \eta_{2} \frac{5 \eta_{1}+3\left(\eta_{2}-\eta_{1}\right) \phi}{5 \eta_{2}-2\left(\eta_{2}-\eta_{1}\right) \phi} ;
\end{aligned}
$$

if $\eta_{1}>\eta_{2}$, the inequality signs must be reversed. It has been demonstrated [17] that for spheres at low concentration $\eta_{\infty}$ equals the lower bound in (39). Consequently one expects for very small $A_{l m}$ 's $\eta_{\infty}$ to be close to this lower bound.

\subsection{Relaxation times of the normal modes of droplets}

In section 4.1 it is made clear that the deformation of the shapes of the droplet can occur in an harmonically oscillating $Y_{20}$-flow. Gradually all the normal modes are excited fully when $\omega$ increases. To find the characteristic times of the normal modes, in a thought experiment an amplitude $A_{l m}^{\prime}$ is applied at time $t_{0}$ on a sphere with radius $a$. When released the amplitude will decrease with some characteristic time $\tau_{l m}$. For the calculation of $\tau_{l m}$ one needs the boundary condition (36). In addition continuity of velocity is required at the surface. The Newtonian fluids are assumed to be incompressible. The Reynolds number is taken to be sufficiently small for the creeping motion equation to be valid in each fluid

$$
\eta \Delta \boldsymbol{u}=\nabla p \text {. }
$$

Here

$$
Y_{l m}^{\theta}(\theta, \phi)=\frac{d}{d \theta} Y_{I m}(\theta, \phi)
$$

Formulae $(41-44)$ can be deduced from the general solution of (40) given by Lamb [18]. Note that radial dependence is only dependent on $l$. For condition (36) $\gamma \operatorname{div} \boldsymbol{n}$ at $r=a$ is needed. It can be calculated that

$$
\gamma \operatorname{div} \boldsymbol{n}=\frac{2 \gamma}{a}+\frac{\gamma}{a^{2}}\left[\frac{l}{2(2 l+3)} A_{l}^{\prime \prime} a^{l+1}+l a^{l-1} C_{l}^{\prime \prime}\right]
$$

$$
\cdot(l+2)(l-1) Y_{l m}(\theta, \phi)
$$

with $A_{l}^{\prime \prime}$ and $C_{l}^{\prime \prime}$ defined by $d A_{l}^{\prime \prime} / d t=A_{l}^{\prime}$ and $d C_{l}^{\prime \prime} / d t=$ $C_{l}^{\prime}$ respectively, while $A_{l}^{\prime}$ and $C_{l}^{\prime}$ are the $A_{l}$ and $C_{l}$ coefficients inside the droplets. Further the velocities and stresses should be finite at $r=0$ and $r=\infty$.

Summarized the boundary conditions (36) at $r=a$ become

for $u\langle r\rangle$ :

$$
\begin{aligned}
& \frac{l}{2(2 l+3)} A_{l}^{\prime} a^{l+1}+l a^{l-1} C_{l}^{\prime} \\
& =\frac{(l+1)}{2(2 l-1)} B_{l} a^{-l}+(-l-1) D_{l} a^{-l-2},
\end{aligned}
$$


for $u\langle\theta\rangle$ :

$$
\begin{gathered}
\frac{(l+3)}{2(2 l+3)(l+1)} A_{l}^{\prime} a^{l+1}+a^{l-1} C_{l}^{\prime} \\
=\frac{(2 l-l)}{2 l(2 l-1)} B_{l} a^{-l}+D_{l} a^{-l-2},
\end{gathered}
$$

for $\sigma\langle r \theta\rangle$ :

$$
\begin{aligned}
& \eta_{2}\left[\frac{l(l+2)}{(l+1)(2 l+3)} A_{l}^{\prime} a^{l+1}+(2 l-2) a^{l-1} C_{l}^{\prime}\right] \\
& \quad=\eta_{1}\left[\frac{(l-1)(l+1)}{l(2 l-1)} B_{l} a^{-l}+(-2 l-4) D_{l} a^{-l-2}\right],
\end{aligned}
$$

for $\sigma\langle r r\rangle$ :

$$
\begin{aligned}
& \eta_{2}\left[\frac{l^{2}-l-3}{2 l+2} A_{l}^{\prime} a^{+1}+2 l(l-1) a^{l-1} C_{l}^{\prime}\right] \\
& \quad+\frac{\gamma}{a}(l-2)(l-1)\left[\frac{l}{2(2 l+3)} a^{l+1} A_{l}^{\prime \prime}+l a^{l-1} C_{l}^{\prime \prime}\right] \\
& \quad=\eta_{1}\left[\frac{l^{2}+3 l-1}{1-2 l} B_{l} a^{-l}+2(l+1)(l+2) D_{l} a^{-l-2}\right]
\end{aligned}
$$

and

$$
p_{0}=p_{0}^{\prime}-\frac{2 \gamma}{a}
$$

at rest.

The solutions of $A_{l}^{\prime}, C_{l}^{\prime}, B_{l}$ and $D_{l}$ for this set of linear differential equations can easily be found substituting for the unknowns their amplitudes at time $t_{0}$ times $\exp \left[\left(t-t_{0}\right) / \tau_{l}\right]$. The set of equations has a solution if the determinant of the coefficients is zero. After some calculations it turns out that

$$
\tau_{l}=\frac{a \eta_{1}}{\gamma} \frac{\left[E 2(l+2)(l-1)+\left(2 l^{2}+1\right)\right]\left[E\left(2 l^{2}+4 l+3\right)+2 l(l+2)\right]}{l(l+1)(l+2)(l-1)(2 l+1)[E+1]}
$$

with $E=\eta_{2} / \eta_{1}$, and $l \geqq 2$.

\subsection{Determination of the emulsion relaxation strengths $G_{l}$}

For $\omega \rightarrow \infty$ all the contributions $G_{l, m}$ to $G^{\prime}$ are excited and the direct influence of $\gamma$ on the viscosity is zero (see section 4.1). The deformation gradient in the radial direction $\varepsilon^{\prime} \sqrt{5 / 4 \pi}$ at the surface is no longer dependent on $l, m$ and will be denoted as $\varepsilon_{\infty}$. The factor $\sqrt{5 / 4 \pi}$ arises from the fact that for a chosen
$\langle\boldsymbol{D}\rangle$ (namely (9)) in polar coordinates the magnitude of the rate of strain $\dot{\varepsilon}$ is the coefficient of the $P_{2}(\cos \theta)$ Legendre polynomial and not of the $Y_{2,0}$ spherical harmonic (32).

To calculate the $G_{l}$ 's a relation is needed between the local deformation gradient $\varepsilon_{\infty}$ in the radial direction at the surface and the average applied deformation gradient $\varepsilon$, because the latter is involved in the macroscopically stored energy per droplet (for a pure staining motion)

$$
\frac{3}{2} G_{l m} \varepsilon^{2} \frac{1}{n}
$$

with $n$ the number of droplets per unit volume.

$$
\begin{aligned}
& \text { Now } \\
& \lim _{\omega \rightarrow \infty}\left\langle\boldsymbol{\varepsilon}_{p}\right\rangle \equiv \lim _{\omega \rightarrow \infty} \frac{1}{2 V_{p}} \int_{\partial V_{p}}(\boldsymbol{\xi} \boldsymbol{n}+\boldsymbol{n} \boldsymbol{\xi}) d S
\end{aligned}
$$

where $\xi$ is the displacement vector and

$$
\varepsilon=\lim _{V \rightarrow \infty} \frac{1}{2 V} \int_{\partial V}(\boldsymbol{\xi} \boldsymbol{n}+\boldsymbol{n} \boldsymbol{\xi}) d S=\varepsilon\left[\begin{array}{ccc}
-1 / 2 & 0 & 0 \\
0 & -1 / 2 & 0 \\
0 & 0 & 1
\end{array}\right]
$$

In a harmonic flow

$$
\begin{aligned}
\lim _{\omega \rightarrow \infty}\left(i \omega \boldsymbol{\varepsilon}_{P}\right\rangle & =\lim _{\omega \rightarrow \infty}\left\langle\boldsymbol{D}_{p}\right\rangle \\
& =\lim _{\omega \rightarrow \infty} \frac{1}{2 V_{p}} \int_{\delta V_{p}}(\boldsymbol{u} \boldsymbol{n}+\boldsymbol{u}) d S
\end{aligned}
$$

for which the linear part should be (38) proportional to

$$
\langle\boldsymbol{D}\rangle=i \omega \varepsilon \text {. }
$$

Apart from the fluctuations the radius $R$ can be written as

$$
R=a\left[1+\varepsilon_{\infty} P_{2}(\cos \theta)\right] .
$$

Consequently in first approximation, using that $\lim _{\omega \rightarrow \infty}\left\langle\boldsymbol{D}_{p}\right\rangle$ is proportional to (55), and using (54) and (58) it holds that

$$
\lim _{\omega \rightarrow \infty}\left\langle\varepsilon_{p}\right\rangle=\varepsilon_{\infty}\left[\begin{array}{ccc}
-1 / 2 & 0 & 0 \\
0 & -1 / 2 & 0 \\
0 & 0 & 1
\end{array}\right] .
$$


For the linear part of $\langle\boldsymbol{\sigma}\rangle$ proportional to the viscosity

$$
\begin{aligned}
\lim _{\omega \rightarrow \infty}\langle\boldsymbol{\sigma}\rangle= & (\text { pressure term })+2 \eta_{1}\langle\boldsymbol{D}\rangle \\
& +\lim _{\omega, V \rightarrow \infty} \frac{1}{V}\left(\eta_{2}-\eta_{1}\right) \int_{\partial V_{p}}(\boldsymbol{u} \boldsymbol{n}+\boldsymbol{n} \boldsymbol{u}) d S \\
= & \text { (pressure term })+2 \eta_{\infty}\langle\boldsymbol{D}\rangle .
\end{aligned}
$$

Apparently

$$
\lim _{\omega \rightarrow \infty}\left\langle\boldsymbol{D}_{p}\right\rangle=\frac{\eta_{\infty}-\eta_{1}}{\phi\left(\eta_{2}-\eta_{1}\right)}\langle\boldsymbol{D}\rangle
$$

and using $(56,57,59,60)$ one finds

$$
\varepsilon_{\infty}=\frac{\eta_{\infty}-\eta_{1}}{\phi\left(\eta_{2}-\eta_{1}\right)} \varepsilon
$$

The energy stored in the extra deformation for a mode is for small amplitudes equal to

$$
W_{\gamma, l, m}^{\prime}-W_{\gamma, l, m}=\frac{1}{2} \gamma a^{2}(l-1)(l+2)\left(A_{l m}^{\prime 2}-A_{l m}^{2}\right) \text {. }
$$

For $A_{l m}^{2}$ its expression given implicitely in (31) has to be inserted. Then a few points can be taken into account: the amplitudes $A_{l m}$ are equally probable positive or negative $\left(\left\langle A_{l m}\right\rangle=0\right)$ and not correlated if indices are different $\left(\left\langle A_{l m} A_{l^{\prime} m}\right\rangle=0\right.$ if $\left.l \neq l^{\prime}\right)$.

In a harmonically oscillating flow the linear term in $\varepsilon_{l m}^{\prime}$ (representing the asymmetry term in the energy for a predeformed surface when deformed with $1+\varepsilon^{\prime} Y_{20}$ and $1-\varepsilon^{\prime} Y_{20}$ ) will average out and be omitted here. This omission is consistent with the assumption that macroscopically the material is not predeformed, see (53).

If

$$
\begin{aligned}
& F_{k^{\prime}}=\sqrt{\frac{5}{4 \pi} \frac{\left(2 k^{\prime}+1\right)}{(2 l+1)}} \text { and } \\
& C_{k^{\prime} m}=C\left(k^{\prime} 2 m 0 \mid k^{\prime} 2 l m\right)
\end{aligned}
$$

the result is

$$
\begin{aligned}
& \left\langle W_{\gamma, l, m}^{\prime}-W_{\gamma, l, m}\right\rangle=\frac{1}{2} \gamma a^{2}(l-1)(l+2) \varepsilon_{l m}^{2} \\
& \cdot\left[\delta_{l 2} \delta_{m 0}+\sum_{k^{\prime}=l-2}^{l+2} F_{k^{\prime}}^{2} C_{k^{\prime} m}^{2} C_{k^{\prime} 0}^{2}\left\langle A_{k^{\prime} m}^{2}\right\rangle\right]
\end{aligned}
$$

Using eqs. $(31,53)$ and $(62)$ with $\varepsilon_{\infty}=\sqrt{\frac{5}{4 \pi}} \varepsilon_{l m}^{\prime}$ $G_{l m}=\left[\frac{1}{2} \gamma a^{2}(l-1)(l+2) \delta_{l 2} \delta_{m 0}\right.$

$$
\left.+\frac{1}{2} k T \sum_{k^{\prime}=l-2}^{l+2} F_{k^{\prime}}^{2} C_{k^{\prime} m}^{2} C_{k^{\prime} 0}^{2}\right] \cdot \frac{2}{5} \frac{\phi}{a^{3}}\left(\frac{\eta_{\infty}-\eta_{1}}{\phi\left(\eta_{2}-\eta_{1}\right)}\right)^{2} .
$$

Note that here still $l$ and $k^{\prime} \geqq 2$ and $G_{l}$ in (2) is

$$
G_{l}=\sum_{m=-l}^{l} G_{l m} .
$$

Considering the Clebsch-Gordan coefficients it turns out that

$C(l-1200) \mid l-12 l 0)=C(l+1200 \mid l+12 l 0)=0$.

The remaining relevant coefficients are [19]:

$C_{l-2 m}=\sqrt{\frac{3(l-m)(l-1-m)(l+m)(l-1+m)}{2(2 l-3)(2 l-1) l(l-1)}}$,

$C_{l m}=\frac{3 m^{2}-l(l+1)}{\sqrt{(2 l-1) l(l+1)(2 l+3)}}$,

$C_{l+2 m}=\sqrt{\frac{3(l+2-m)(l+1-m)(l+2+m)(l+1+m)}{2(l+1)(l+2)(2 l+3)(2 l+5)}}$

\subsection{Discussion of the emulsion results}

In the previous sections the relaxation times $\tau_{l}$ and strengths $G_{l m}$ of the complex viscosity $\eta^{*}$ for statistically fluctuating droplets have been derived. For low volume fractions and $T=0$ the well-known $G^{*}$ derived by Oldroyd [13] is found if the relevant $\eta_{\infty}$ given by the lower bound of (39) for $\phi \rightarrow 0$ is used. The steady-state viscosity is given by

$$
\eta_{O}=\eta_{\infty}+\sum_{l} G_{l} \tau_{l}
$$

Striking is the influence of the ratio $k T / 4 \pi a^{2} \gamma$. This is shown in figures 1 and 2. The ratio $k T / 4 \pi a^{2} \gamma$ is chosen as 0.02 and 0.2 respectively. The path of $\eta^{*}$ can change appreciably due to shape fluctuations of the droplets. Obviously the $\eta_{o}$ viscosity can be larger than the Taylor value due to deviations from the spherical shape.

The validity of the derived formulae is limited, because $\left|A_{l m}\right|$ should be much smaller than unit. Using eq. (31) this implies that

$$
\tau_{2} \approx \frac{a \eta}{\gamma} \ll \frac{(l-1)(l+2)}{2 k T} a^{3} \eta,
$$

thus

$$
\tau_{2} \ll \frac{2 a^{3} \eta}{k T}<\frac{a^{2}}{D_{0}}
$$

where $D_{0}=k T / 6 \pi \eta a$ is the diffusion coefficient of a sphere with radius $a$. 


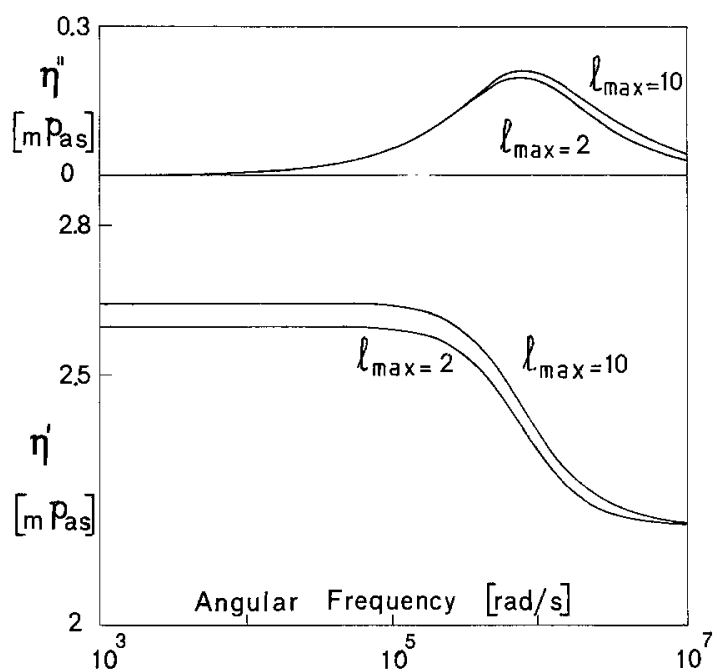

Fig. 1. Complex viscosity $\eta^{*}=\eta^{\prime}-i \eta^{\prime \prime}$ of the thermally fluctuating droplet model as a function of the angular frequency with two $l_{\max }$ parameter values for $4 \pi \gamma a^{2}=50 \mathrm{kT}$

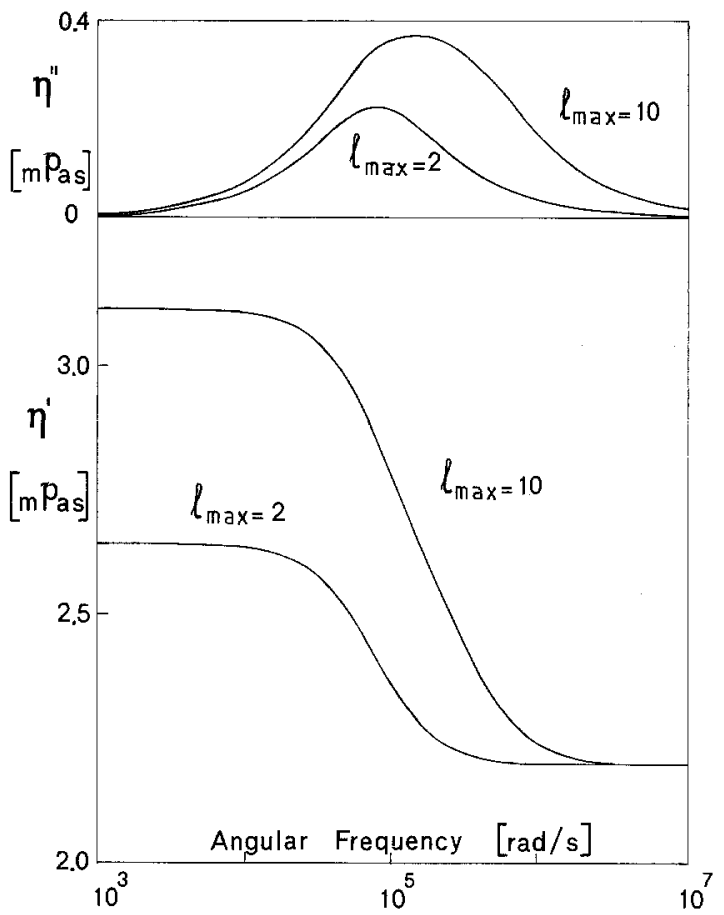

Fig. 2. Complex viscosity $\eta^{*}=\eta^{\prime}-i \eta^{\prime \prime}$ of the thermally fluctuating droplet model as a function of the angular frequency with two $l_{\max }$ parameter values for $4 \pi \gamma a^{2}=5 k T$

This is a satisfactory condition because to be in the linear viscoelastic range $a^{2} / D \ll \dot{\varepsilon}^{-1}$ should hold (see [20]) and the longest relaxation time should be much smaller than the typical time $\dot{\varepsilon}^{-1}$ characterising the flow. The inequalities in (71) imply that the derived formulae are also valid in a shear field since the

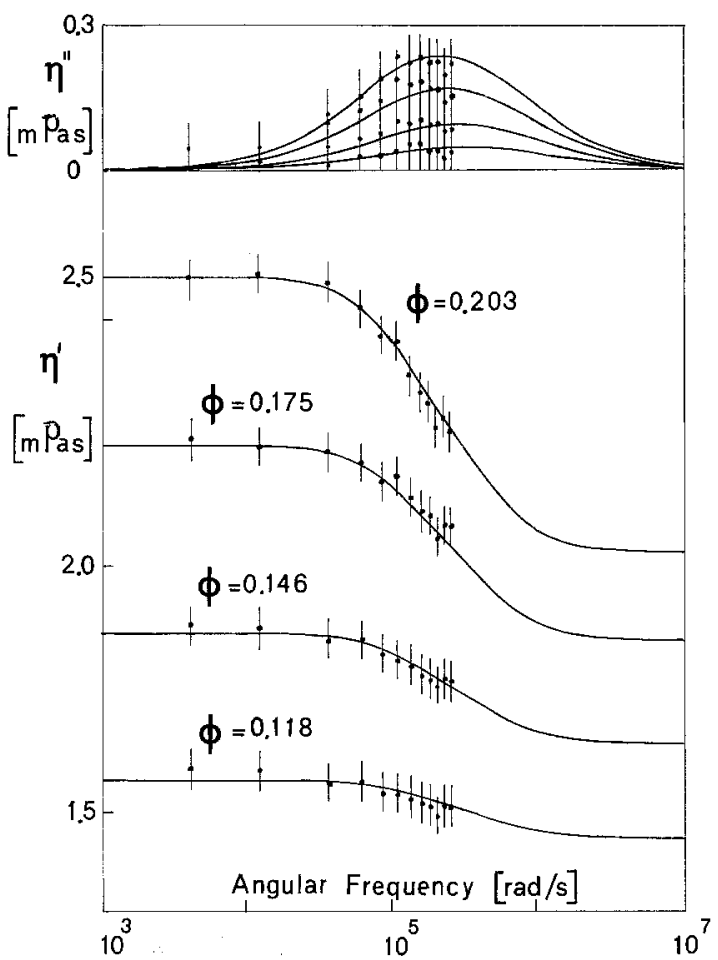

Fig. 3. Complex viscosity $\eta^{*}=\eta^{\prime}-i \eta^{\prime \prime}$ of a non-ionic microemulsion as a function of the angular frequency and with the volume fraction as parameter value fitted with the thermally fluctuating droplet model. Fitting parameters $\gamma=22 \pm 2 \cdot 10^{6}$ $\mathrm{N} / \mathrm{m}, \eta_{3}=17 \pm 3 \mathrm{mPa} \mathrm{s}, a=10 \mathrm{~nm}$ and $l_{\max }=8$

rotational time for the shear field is than much larger than $\tau_{2}$. In figure 3 we applied the results to a microemulsion. Microemulsions are optically isotropic waterin-oil or oil-in-water liquid systems, which form spontaneously in the presence of a surfactant usually in combination with a cosurfactant (alcohol). For a certain overall composition and temperature the mixture becomes a dispersion of tiny oil droplets in water. It turned out [7] that for a particular microemulsion the amount of water can be changed without change of structure if a certain ratio of oil-surfactant is kept constant. In figure 3 such a dilution series, with volume fractions $\phi=0.203,0.175,0.146,0.118$, is shown for a mixture of $\mathrm{n}$-hexane, water and NNP7 (polyoxyethylated (7)-nonylphenolether) with a constant $\mathrm{wt} / \mathrm{wt}$ ratio of 1.2 for NNP7/hexane. The series has been measured with a nickel tube resonator [6]. The radius of the droplets, estimated by dynamic lightscattering and a geometrical model, is about $10 \mathrm{~nm}$. Obviously the viscoelastic effects are small. The error boundaries are about $2 \%$ of the $\eta^{\prime}$-value. This is an empirical value including systematic and statistical contributions. Comparing the $\eta^{\prime}$-values for $\omega \rightarrow 0$ with 
the steady-state values of $\eta$ for hard spheres in the linear range [20] the $\eta^{\prime}$-values appear to be slightly higher. Such a phenomenon can be explained by the present model where the increase of the viscosities with respect to those of hard spheres is due to small deformations of spherical droplets, characterized with a constant interfacial tension $\gamma$. Since we deal here with a high volume concentration adjustments of variables in the formulae are necessary. Since the effect of single particles is pursued one can suppose that the effect of other droplets surrounding a considered droplet is the change of the effective viscosity outside the droplet surface. Thus some effective $\eta_{\text {eff }}$ should replace $\eta_{1}$ in the $\tau_{l}$ 's. Here it is chosen to be $\frac{1}{2}\left(\eta_{O}+\eta_{\infty}\right)$. Secondly the droplet surface is mainly composed of surfactant molecules in a high concentration. This gives an interface with a viscosity $\eta_{3}$ of $10 \mathrm{mPas}$ or higher. Consequently the factor $\left(\eta_{\infty}-\eta_{1}\right) / \phi\left(\eta_{2}-\eta_{1}\right)$ has to be replaced by a factor close to $\left(\eta_{\infty}-\eta_{1}\right) / \eta_{3} \phi_{s}$ with $\phi_{s}$ the volume fraction of this surfactant layer.

If these modifications with two free parameters $\eta_{\mathrm{eff}}$ in $\tau_{l}$ and $\eta_{3} \phi_{s}$ are incorporated a fit is possible as shown in figure 3 . The maximum $l$-value is according to Ljunggren and Eriksson [21]

$$
2 \sqrt{\pi} \frac{a}{\lambda_{\min }}-1 .
$$

Here $\lambda_{\min }$ is chosen as 6 times the diameter of the NNP7 molecule resulting in $l_{\max }=8$.

In this fit $\eta_{\infty}$ is a free parameter for all volume fractions. The interfacial voluminae $\phi_{s}$ are estimated on the basis of the measured specific density and the weighed amount of NNP7. The interface viscosity $\eta_{3}$ is chosen to be the same for all microemulsions but otherwise to be fitted. Changing iteratively $\eta_{\infty}$ and $\eta_{3}$ finally the interfacial tension $\gamma$ is deduced under the condition that it is the same for different volume fractions. It has been found that $\gamma=(22 \pm 2) \cdot 10^{-6}$ $\mathrm{N} \mathrm{m}^{-1}$ and $\eta_{3}=17 \pm 3 \mathrm{mPa}$. The order of magnitude of the derived tension is consistent with the mentioned values in the literature though it has never been measured before for these tiny droplets. The ratio $4 \pi a^{2} \gamma / k T=6.6$. The ensueing structure is that of slightly deformed droplets dispersed in a water continuous medium. This morphology is consistent with current concepts of microemulsions.

\section{References}

1. Rouse PE Jr (1953) J Chem Phys 21:1272

2. Zimm BH (1956) J Chem Phys $24: 269$

3. Oldroyd JG (1953) Proc Roy Soc London Ser A, 218:122

4. Oldroyd JG (1955) Proc Roy Soc London Ser A, 232:567

5. Oosterbroek M, Mellema J, Lopulissa JS (1981) J Colloid Interface Sci 84:27

6. Oosterbroek M, Waterman HA, Wiseall SS, Altena EG, Mellema J, Kip GAM (1980) Rheol Acta 19:497

7. Eshuis A, Mellema J (1984) Colloid Polymer Sci 262:159

8. Blom C, Mellema J (1984) J Disp Sci and Techn 5:193

9. Ferry JD (1969) Viscoelastic properties of polymers. John Wiley \& Sons, Inc, New York

10. Axelrad DR (1970) Adv Molec Relexation Processes 2:41

11. Rayleigh JWS (1945) The theory of sound, vol I. Dover Publications, New York

12. Gross B, Fuoss RM (1956) J Polym Sci 19:39

13. Bird RB, Armstrong RC, Hassager O (1977) Dynamics of polymer liquids, Vol I. John Wiley \& Sons, New York

14. Mijnlieff PF (1984) in: Mena B et al (eds), Advances in rheology vol 1, p 459. Universidad Nacional Autonoma de México, México

15. Cronin JA, Greenberg DF, Telegdi VT. Graduate problems in physics, $\mathrm{p} 257$, University of Chicago

16. Merzbacher E (1963) Quantum Mechanics. John Wiley \& Sons, New York

17. Mellema J, Willemse MWM (1983) Physica 122 A:286

18. Lamb H (1975) Hydrodynamics. Cambridge University Press

19. Condon EU, Shortley EU (1967) The theory of atomic spectra. Cambridge University Press, $p 77$

20. de Kruif CG, Van Iersel EMF, Vrij A, Russel WB (1985) J Chem Phys 83:4717

21. Ljunggren S, Eriksson JC (1984) J Chem Soc Faraday Trans 2, 80:489

(Received March 3, 1987)

Authors' address:

J. Mellema, C. Blom, J. Beekwilder

Rheology Group

Department of Applied Physics

Twente University of Technology

P.O. Box 217

NL-7500 AE Enschede 